Article

\title{
Asymmetry for Symmetry: Right-Hemispheric Superiority in Bi-Dimensional Symmetry Perception
}

\author{
Giulia Prete ${ }^{1, *}$, Mara Fabri ${ }^{2}$, Nicoletta Foschi ${ }^{3}$ and Luca Tommasi ${ }^{1}$ \\ 1 Department of Psychology, Health and Territory, G. d'Annunzio University of Chieti-Pescara, \\ Chieti 66013, Italy; luca.tommasi@unich.it \\ 2 Department of Clinical and Experimental Medicine, Neuroscience and Cell Biology Section, \\ Polytechnic University of Marche, Ancona 60121, Italy; m.fabri@univpm.it \\ 3 Regional Epilepsy Centre, Neurological Clinic, Ospedali Riuniti, Ancona 60126, Italy; \\ n.foschi@ospedaliriuniti.marche.it \\ * Correspondence: giulia.prete@unich.it; Tel.: +39-871-3554216; Fax: +39-871-35542163 \\ Academic Editor: Lesley Rogers \\ Received: 2 April 2017; Accepted: 16 May 2017; Published: 18 May 2017
}

\begin{abstract}
A right-hemispheric superiority has been shown for spatial symmetry perception with mono-dimensional stimuli (e.g., bisected lines). Nevertheless, the cerebral imbalance for bi-dimensional stimuli is still controversial, and the aim of the present study is to investigate this issue. Healthy participants and a split-brain patient (D.D.C.) were tested in a divided visual field paradigm, in which a square shape was presented either in the left or right visual field and they were asked to judge whether a dot was placed exactly in the center of the square or off-center, by using the left/right hand in two separate sessions. The performance of healthy participants was better when the stimuli presented in the left visual field (LVF) were on-center rather than off-center. The performance of D.D.C. was higher than chance only when on-center stimuli were presented in the LVF in the left hand session. Only in this condition did his accuracy not differ with respect to that of the control group, whereas in all of the other conditions, it was lower than the controls' accuracy. We conclude that the right-hemispheric advantage already shown for mono-dimensional stimuli can be extended also to bi-dimensional configurations, confirming the right-hemispheric superiority for spatial symmetry perception.
\end{abstract}

Keywords: perceptual symmetry; cerebral hemispheres; split-brain patient; spatial processing; bi-dimensional stimuli

\section{Introduction}

Symmetry is easily detected by the visual system, and the way in which humans and other animals process visual symmetry is a central issue both in psychology and neuroscience. In fact, several models have been proposed in the attempt to explain how symmetry is detected and analyzed by the brain (e.g., [1-7]). Among the most acknowledged models, the perceptual rules proposed by Gestalt psychologists suggested that our preference for symmetric configurations ("symmetry bias") could be considered as a consequence of the perceptual preference for regularity and balance, compared to randomness and imbalance, by the human visual system. A debated point in this context concerns the possibility that such a regularity is extracted automatically, or it calls attentional processes into play (see [3] for a review). In support of the first point of view, it has been shown that patients with hemispatial neglect ("blind" for the left visual field as a consequence of a right-hemispheric lesion) show a preference for symmetrical arrangements in both visual fields, confirming that preattentive processes are responsible for figure-ground organization [8-10]. Other important results in this context come from those patients who have undergone surgical resection of callosal fibers, in the attempt to 
avoid the spread of epileptic foci between the two cerebral hemispheres, the so called "split-brain patients" [11]. In a series of experiments carried out with a split-brain patient, Funnell, Corballis, and Gazzaniga [12] showed that the right disconnected hemisphere is superior to the left hemisphere in perceptual matching tasks with mirror-reversed stimuli. By presenting stimuli consisting of either color pictures of nameable objects, black-and-white line drawings, or abstract geometrical forms, the authors concluded that the left hemisphere is specialized in pattern recognition, whereas the right hemisphere is specialized in spatial processing (see also [13]). The split-brain patients' literature is strongly linked to that of perceptual symmetry, because a number of authors suggested that at least when talking about vertical symmetry perception, this mechanism is due to the symmetrical morphology of our brain. In other words, it has been suggested that the preferential activation of two homologue areas in the left and right hemispheres is the basis for the automatic detection of symmetry in the physical world. In this "callosal hypothesis", the detection of symmetry may be favored by the activity of two specular areas in the left and right halves of the brain, which are connected by means of the fibers constituting the corpus callosum $[1,2,14-16]$. In this frame, it has been suggested that both the left and the right hemispheres are capable of low-level perceptual processing, and that hemispheric asymmetries arise at later stages of visual processing, in associative areas representing the two sides of visual space [17]. It has to be highlighted, however, that contrasting models have been recently suggested $[18,19]$. The fact that higher order cortical areas are involved in the detection of symmetrical patterns was confirmed in a functional magnetic resonance imaging (fMRI) study [20]: it was shown that independently of the size and the geometrical configuration of the stimuli, as well as independently of the recruitment of attentional control, symmetrical arrangements activated associative visual areas, in particular V3, V4, V7, and lateral occipital areas (for similar results see also [21,22], for a review see [23]).

A right-hemispheric superiority for symmetry detection has been found in healthy participants by means of the divided visual field paradigm. Wilkinson and Halligan [24] presented lines which could be either perfectly divided into two halves (bisected lines) or divided into two asymmetrical segments (misbisected lines), either in the left visual field (LVF) or in the right visual field (RVF), and participants were asked to judge whether each stimulus was symmetrical or asymmetrical. The performance of participants was better in terms of both accuracy and response times when bisected lines, but not misbisected lines, were presented in the LVF, concluding in favor of a right-hemispheric preference for symmetry. Besides the advantage of the right hemisphere in geometrical processing, the authors explained their results also by referring to the differential hemispheric specialization for low and high spatial frequencies. In particular, they concluded that the cerebral asymmetry they found could also be due to the fact that the right hemisphere is more strictly linked than the left hemisphere to the magnocellular visual pathway. This pathway is more sensitive to the low spatial frequencies of the stimuli, which are processed faster than the high spatial frequencies [25], and this could be intended as a further reason for the faster detection of symmetry by the right hemisphere. According to the authors, the short stimulus exposure used in the divided visual field paradigm (tachistoscopic presentation), together with the lateralized presentation of the stimuli (eccentricity), may facilitate the low spatial frequency analysis and thus the right-hemispheric processing. The same right-hemispheric superiority for low spatial frequencies has been confirmed also by means of complex visual stimuli, both in healthy participants and in split-brain patients (e.g., [26-28]). Nevertheless, in the same study Wilkinson and Halligan [24] failed to find a cerebral imbalance in the detection of symmetry when "double axes stimuli" (squares in which a circle could be placed on-center or off-center) were presented, explaining this finding as possibly attributable to the fact that square bisection activates bilateral networks. In a following fMRI study, Wilkinson and Halligan [29] found that the cerebral substrate of the LVF advantage for detecting the presence/absence of symmetry in lines is the right anterior cingulate gyrus. Bertamini and Makin [30] found that symmetry processing induced occipital alpha Event Related Desynchronization (ERD) in the right hemisphere, confirming at the electrophysiological level the stronger right- than left-hemispheric involvement in symmetry detection (see also [31,32]). In another electroencephalographic (EEG) study, Makin and colleagues [33] also showed that the 
Sustained Posterior Negativity is stronger for reflection than for rotation and translation, and that this is true when participants were explicitly required to detect the presence of regularity in the stimuli.

The right-hemispheric causal involvement in symmetry detection has been demonstrated by means of transcranial magnetic stimulation (TMS) studies: Bona and colleagues [34] applied TMS over the left or right lateral occipital cortex while participants were asked to distinguish symmetrical from asymmetrical random dot patterns. The authors found that both hemispheres are involved in the task, but that the right-hemispheric stimulation leads to a stronger disruption of symmetry detection with respect to the left-hemispheric stimulation (see also [35]). TMS was also exploited together with an adaptation paradigm, revealing that when applied between adaptation and test stimuli, TMS applied over the dorsolateral extrastriate cortex, but not over V1/V2, reduced adaptation effects to dot patterns [36].

Only in one study among those reviewed above, has the cerebral asymmetry for double axes figures been investigated [24], and the authors failed to find significant differences in the ability to detect symmetry between the left and right hemispheres. Starting from this evidence, the main aim of the present study is to further assess this issue both in healthy participants and in a split-brain patient. In particular, in a divided visual field paradigm, participants were presented with stimuli consisting of a square containing a dot placed either in its exact center or slightly off-center. They were asked to judge whether the circle was/was not placed exactly in the center of the square. We hypothesized that, as found in previous works with single axis stimuli, a LVF superiority may be observed also for double axes stimuli, starting from the several studies of a right-hemispheric superiority for symmetry detection. To this aim, we almost quadrupled the number of healthy participants with respect to the study of Wilkinson and Halligan (from 12 to 44 participants), we further shortened the tachistoscopic presentation time (from $170 \mathrm{~ms}$ to $150 \mathrm{~ms}$ ), and we also tested a complete callosotomy patient in order to obtain data from each surgically disconnected hemisphere. Additionally, in contrast to Wilkinson and Halligan, we asked participants to take part in two separate sessions, differing from one another in the hand used to respond, in order to consider the hand of the response as a further within-subject factor in the statistical design. Specifically, we did not expect to find differences in healthy participants according to the hand used to respond (the use of one hand does not allow us to test the unilateral responses in the intact brain), but we expected that the use of one hand in the split-brain patient would ensure the contralateral hemispheric involvement (due to the contralateral organization of the motor pathways; e.g., [37-39]). By using these changes, we expected to confirm that the performance is better when symmetrical (on-center) stimuli were presented in the LVF, both in healthy participants and-importantly-in the patient. The right-hemispheric superiority for spatial processing in split-brain patients has already been shown [12,13], but it has not been investigated for symmetry detection. In a study involving two split-brain patients, including the patient tested here, Corballis et al. [40] found a right-hemispheric superiority when patients were asked to distinguish between canonical and mirror-reversed letters ( $\mathrm{F}$ and $\mathrm{R}$ ), concluding that this task depends on matching to an exemplar (the canonical oriented letter), for which the right-hemisphere is dominant (as opposed to the left-hemispheric superiority in letter naming). In contrast to that study, in the present study we presented geometrical shapes, for which no comparison with a "model" is required, and thus we aimed at investigating the pure hemispheric imbalance in symmetry detection.

\section{Materials and Methods}

\subsection{Participants}

D.D.C. is an Italian male patient suffering from medically intractable epilepsy, who has had the corpus callosum (CC) surgically sectioned in the attempt to avoid the spread of epileptic foci between the cerebral hemispheres. He underwent the first partial section of CC in 1994, when he was 18-year old, and the complete section in 1995; the anterior commissure was also resected (see Figure 1). D.D.C. was 38 years old at the time of the test, his postoperative IQ was 83 , and his laterality 
quotient was +40 (for more details, see [41]). The patient declared that he wrote with his left hand until he was 10, and then he was forced to use the right hand. D.D.C. is free from perceptual or motor impairments, and he has intact linguistic skills in both hemispheres [41]. He was tested at the Epilepsy Center of the Polytechnic University of Marche (Torrette of Ancona), during a pause between routine neurological examinations.

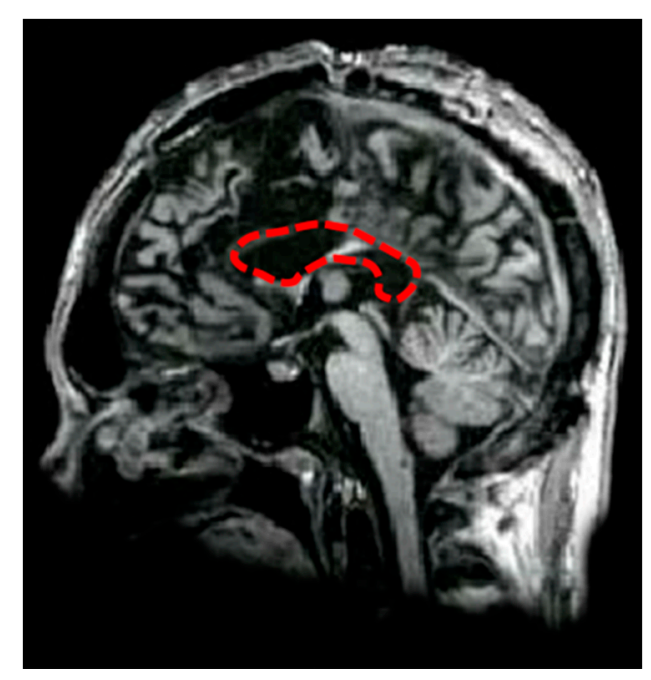

Figure 1. Midsagittal MRI of patients: the figure shows D.D.C.'s brain, showing the complete absence of callosal fibers (in the area delimited by the red-dashed line).

The control group was composed of 44 healthy volunteers (22 female; age: $\mathrm{M}=25.5 \pm 0.89$ ). All were right-handed as assessed by the Edinburgh Handedness Inventory ([42]; $M=67.31 \pm 2.28$ ), had normal or corrected-to-normal vision, and none of them had any neurological or psychiatric history. These participants were tested at the Psychobiology Laboratory of the University of Chieti.

Informed consent was obtained from all participants prior to the experiment and the experimental procedures were conducted in accordance with the guidelines of the Declaration of Helsinki.

\subsection{Stimuli}

Stimuli consisted of a square designed by means of the software Microsoft PowerPoint 2007 (Microsoft Corp., Redmond, WA, USA). The square shape had black contours and it encompassed a white area measuring $7.6 \times 7.6 \mathrm{~cm}$ (width $\times$ height; $4.2 \times 4.2$ degrees of visual angle, seen at a distance of $72 \mathrm{~cm}$, on a screen with a resolution of $1280 \times 768$ pixels). The perimeter of the figure measured $0.3 \mathrm{~mm}$ in thickness. A black circular dot with a diameter measuring $0.9 \mathrm{~cm}$ was placed within the square. The dot was placed perfectly in the center of the square in half of the stimuli (On-Center condition), whereas in the other half it was placed $3 \mathrm{~mm}\left(0.18^{\circ}\right.$ of the visual angle) away from the center (Off-Center condition: 25\% above, 25\% below, 25\% left, and 25\% right, with respect to the center of the square).

\subsection{Procedure}

Each trial started with a vertical red line, measuring $2 \mathrm{~mm}$ in thickness and $16 \mathrm{~cm}$ in height, presented in the center of a white screen for $1000 \mathrm{~ms}$. In the following $150 \mathrm{~ms}$ the red line remained visible, and a stimulus was presented in the center of the left or right half of the screen (the center of the square was placed at $3.7^{\circ}$ of the visual angle from the center of the screen, with the innermost edge placed at $1.6^{\circ}$ of the visual angle). Finally, a white screen was presented until the participant gave the response, and then the next trial started. 
Each participant took part into two sessions, each composed of 96 trials. In each session, the stimulus was presented 48 times in the left visual field (LVF) and 48 times in the right visual field (RVF). For each visual field, 24 trials consisted of the square containing the dot in the center (On-Center condition), and 24 trials consisted in the square containing the dot moved away from the center (Off-Center condition, with 6 trials for each position: up, down, left, right; see Figure 2). The presentation order of the trials was randomized within and among participants.

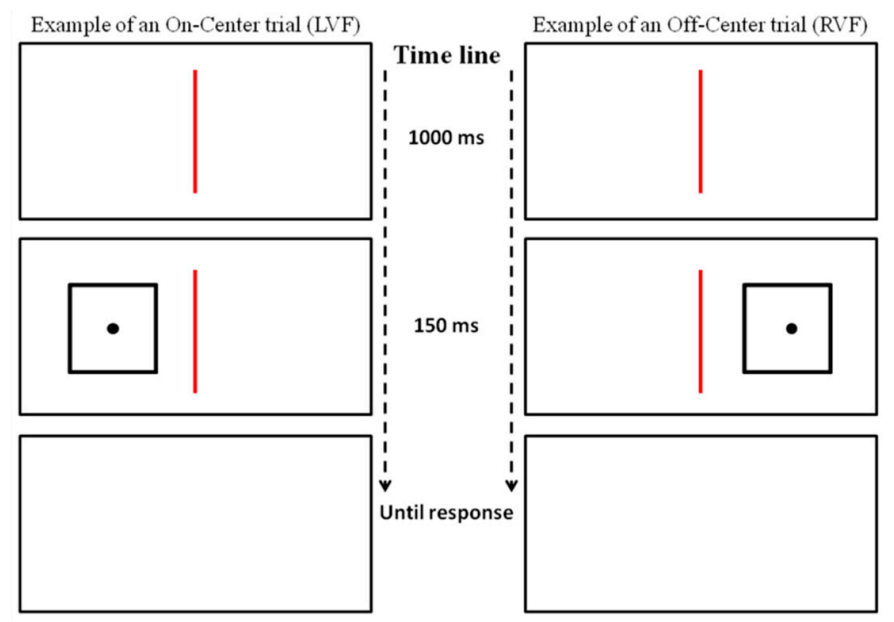

Figure 2. An example of (1) an On-Center trial, in which a symmetrical stimulus is presented in the Left Visual Field (LVF, left panel) and (2) an Off-Center trial, in which an asymmetrical stimulus is presented in the Right Visual Field (RVF, right panel).

Participants were tested in isolation. Before the beginning of the experiment, 4 trials were presented to allow the participants to become familiar with the task. They were asked to maintain their gaze in the center of the screen for the whole task, on the red line (a red line was used instead of the most conventional central fixation cross, in order to avoid possible cues concerning the exact center of the screen on the horizontal plane), and to evaluate in each trial the position of the dot inside the square. Specifically, they were asked to press one key when the dot was perfectly placed in the center of the square, and a different key when the dot was not in the center. They were also informed that the dot position could be a few millimeters away from the exact center of the square, in any direction (up, down, left, right). In the two experimental sessions, participants were required to carry out the task using either the left hand or the right hand, and the order of the two sessions was balanced among the participants. D.D.C. started with the right hand session.

The paradigm was controlled by means of E-Prime software (Psychology Software Tools Inc., Pittsburgh, PA, USA), and lasted about $15 \mathrm{~min}$.

\section{Results}

\subsection{Control Group}

Data were analyzed by means of an analysis of variance (ANOVA), in which the Inverse Efficiency Score (IES) was the dependent variable. The IES was calculated by dividing the response times obtained in the correct responses by the proportion of correct responses in each condition. Reaction times were excluded when they were lower than $150 \mathrm{~ms}$ and higher than $1500 \mathrm{~ms}$ (3.11\% of the trials). In a first ANOVA, Order of sessions (first session: Left hand, Right hand) and Sex of participants (Female, Male) were considered as between-subjects factors, and Hand of response (Left, Right), Visual field of presentation (Left, Right), and Condition (On-Center, Off-Center) were considered as within-subjects factors. Neither Order of sessions, nor Sex of participants were significant, nor did they interact with the other factors, thus they were excluded from the main analysis. 
The ANOVA was carried out considering three within-subject factors: Hand of response (Left, Right), Visual field of presentation (Left, Right), and Condition (On-Center, Off-Center), and the IES was considered as the dependent variable. When required, the Duncan test was used for post-hoc comparisons. The main effect of Condition was significant $\left(F_{(1,43)}=4.060, p=0.050, \eta_{p}^{2}=0.09\right)$, showing that the performance of participants was better in the On-Center condition (922.76 \pm 101.46$)$, than in the Off-Center condition (1423.06 \pm 132.46$)$. Importantly, the interaction between Condition and Visual field was significant $\left(F_{(1,43)}=6.196, p=0.017, \eta_{p}{ }^{2}=0.13\right)$. Post-hoc comparisons revealed that when stimuli were presented in the LVF, the performance of participants was better in the On-Center condition than in the Off-Center condition $(p<0.001)$. Moreover, in the On-Center condition, the performance was better when stimuli were presented in the LVF than in the RVF, and the opposite was true for the Off-Center condition, even if both comparisons failed to reach statistical significance (On-Center: $p=0.088$; Off-Center: $p=0.083$; Figure 3). Other main effects and interactions were not significant.

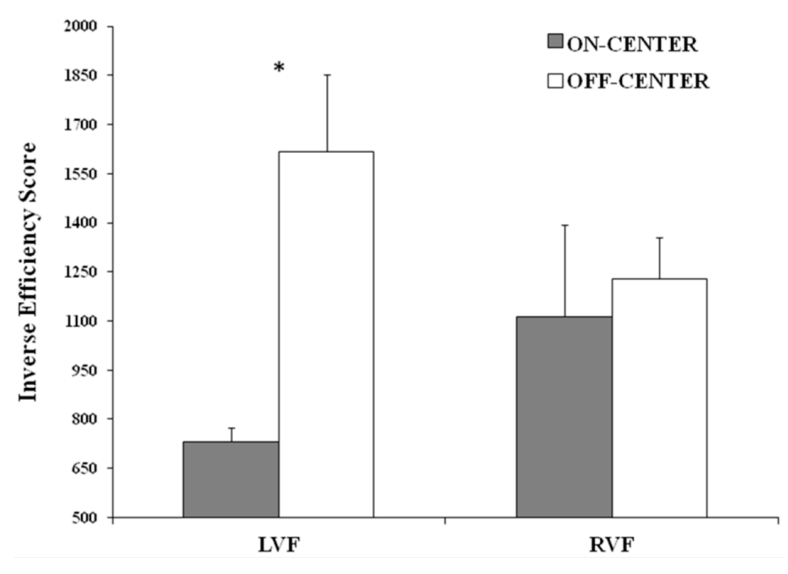

Figure 3. Interaction between the Visual field of presentation (Left, Right) and Condition (On-Center, Off-Center) on the Inverse Efficiency Score (response times of the correct responses divided by the proportion of correct responses) in healthy participants. Bars represent standard errors and the asterisk shows the significant comparison.

\subsection{D.D.C.}

The results of D.D.C. were analyzed by using a binomial distribution analysis and chi-square tests (as in [39]). The binomial distribution was computed considering the frequency of correct responses in each condition (Hand of response $\times$ Visual field $\times$ Condition). The results showed that the patient's responses were given at the chance level (50\%) in any condition in the Right hand session, and in the On-Center condition-RVF in the Left hand session. In the Left hand session, his performance was significantly below the chance level for the Off-Center condition in both LVF and RVF, whereas it was significantly above the chance level only in the On-Center condition-LVF (Table 1).

Table 1. The frequencies of correct responses of patient D.D.C., and respective probabilities in the binomial distribution, in the Left hand session (upper panel) and in the Right hand session (lower panel), for stimuli presented in the Left Visual Field (LVF) and in the Right Visual Field (RVF), in the On-Center and Off-Center condition. Significant results are represented in bold.

\begin{tabular}{|c|c|c|c|c|c|}
\hline \multirow[t]{2}{*}{ Session } & \multirow[t]{2}{*}{ Results } & \multicolumn{2}{|c|}{ LVF } & \multicolumn{2}{|c|}{ RVF } \\
\hline & & On-Center & Off-Center & On-Center & Off-Center \\
\hline \multirow{2}{*}{ Left hand } & Correct responses & 20 & 6 & 13 & 3 \\
\hline & Binomial: $p$ & $<0.001$ & 0.008 & 0.149 & $<0.001$ \\
\hline \multirow{2}{*}{ Right hand } & Correct responses & 11 & 9 & 13 & 12 \\
\hline & Binomial: $p$ & 0.149 & 0.078 & 0.149 & 0.161 \\
\hline
\end{tabular}


Chi-square tests were used to compare the frequency of correct responses in the LVF vs RVF, as well as in the On-Center vs Off-Center conditions, in each session (Left hand, Right hand). Only the comparison between the On-Center and Off-Center conditions in the Left hand session was significant, showing that the patient correctly categorized the stimuli more frequently in the On-Center condition (Table 2). Thus, in order to better investigate this effect, chi-square tests were also computed for all of the interactions between VF and Condition. In the Right hand session, the comparisons were not significant. In the Left hand session, the comparisons between LVF and RVF were not significant either for the On-Center or for the Off-Center conditions, but D.D.C. categorized stimuli better in the On-Center condition than in the Off-Center condition, when presented with both in the LVF $\left(\chi^{2}=7.54\right.$, $p=0.006)$ and in the RVF $\left(\chi^{2}=6.25, p=0.012\right.$; see Table 2$)$.

Table 2. The frequencies of correct responses of patient D.D.C., Chi-square values and respective significance levels in the Left hand session and in the Right hand session, for stimuli presented in the Left Visual Field (LVF) and in the Right Visual Field (RVF), in the On-Center and Off-Center condition, as well as their interactions. Significant results are represented in bold.

\begin{tabular}{|c|c|c|c|c|c|c|c|c|}
\hline \multirow[t]{2}{*}{ Results } & \multicolumn{4}{|c|}{ LEFT HAND } & \multicolumn{4}{|c|}{ RIGHT HAND } \\
\hline & LVF & RVF & On-Center & Off-Center & LVF & RVF & On-Center & Off-Center \\
\hline Correct responses & 26 & 16 & 33 & 9 & 20 & 25 & 24 & 21 \\
\hline $\mathrm{Chi}^{2}$ & \multicolumn{2}{|c|}{2.38} & \multicolumn{2}{|c|}{13.71} & \multicolumn{2}{|c|}{0.55} & \multicolumn{2}{|c|}{0.2} \\
\hline \multirow[t]{3}{*}{$p$} & \multirow{2}{*}{\multicolumn{2}{|c|}{$\begin{array}{c}0.122 \\
\text { On-Center }\end{array}$}} & \multirow{2}{*}{\multicolumn{2}{|c|}{$\begin{array}{c}<0.001 \\
\text { Off-Center }\end{array}$}} & \multirow{2}{*}{\multicolumn{2}{|c|}{$\begin{array}{c}0.456 \\
\text { On-Center }\end{array}$}} & \multirow{2}{*}{\multicolumn{2}{|c|}{$\begin{array}{c}0.655 \\
\text { Off-Center }\end{array}$}} \\
\hline & & & & & & & & \\
\hline & LVF & RVF & LVF & RVF & LVF & RVF & LVF & RVF \\
\hline Correct responses & 20 & 13 & 6 & 3 & 11 & 13 & 9 & 12 \\
\hline $\mathrm{Chi}^{2}$ & \multicolumn{2}{|c|}{1.485} & \multicolumn{2}{|c|}{1} & \multicolumn{2}{|c|}{0.167} & \multicolumn{2}{|c|}{0.428} \\
\hline$p$ & \multirow{2}{*}{\multicolumn{2}{|c|}{0.223}} & \multirow{2}{*}{\multicolumn{2}{|c|}{$\begin{array}{l}0.317 \\
\text { RVF }\end{array}$}} & 0.6 & & & \\
\hline & & & & & \multicolumn{2}{|c|}{ LVF } & \multicolumn{2}{|c|}{ RVF } \\
\hline & On-Center & Off-Center & On-Center & Off-Center & On-Center & Off-Center & On-Center & Off-Center \\
\hline Correct responses & 20 & 6 & 13 & 3 & 11 & 9 & 13 & 12 \\
\hline $\mathrm{Chi}^{2}$ & \multicolumn{2}{|c|}{7.54} & \multicolumn{2}{|c|}{6.25} & \multicolumn{2}{|c|}{0.2} & \multicolumn{2}{|c|}{0.04} \\
\hline$p$ & \multicolumn{2}{|c|}{0.006} & \multicolumn{2}{|c|}{0.012} & \multicolumn{2}{|c|}{0.655} & \multicolumn{2}{|c|}{0.841} \\
\hline
\end{tabular}

\subsection{Control Group vs. D.D.C.}

The mean percentage of correct responses for each condition obtained in the control group was compared with the percentage of D.D.C.'s correct responses, by means of exact $t$-tests, using the percentage of responses by the patient as a reference value for each session (Left hand and Right hand), separately (as in $[39,43])$.

The results showed that healthy participants gave more correct responses than D.D.C. in all conditions and in both sessions (for all comparisons: $3.39<t_{(43)}<18.21, p \leq 0.001$ ), with the exception of the On-Center condition-LVF in the Left hand session, where no difference between the patient's performance and the control group's performance was observed $\left(t_{(43)}=-1.23, p=0.225\right.$; Figure 4$)$. 


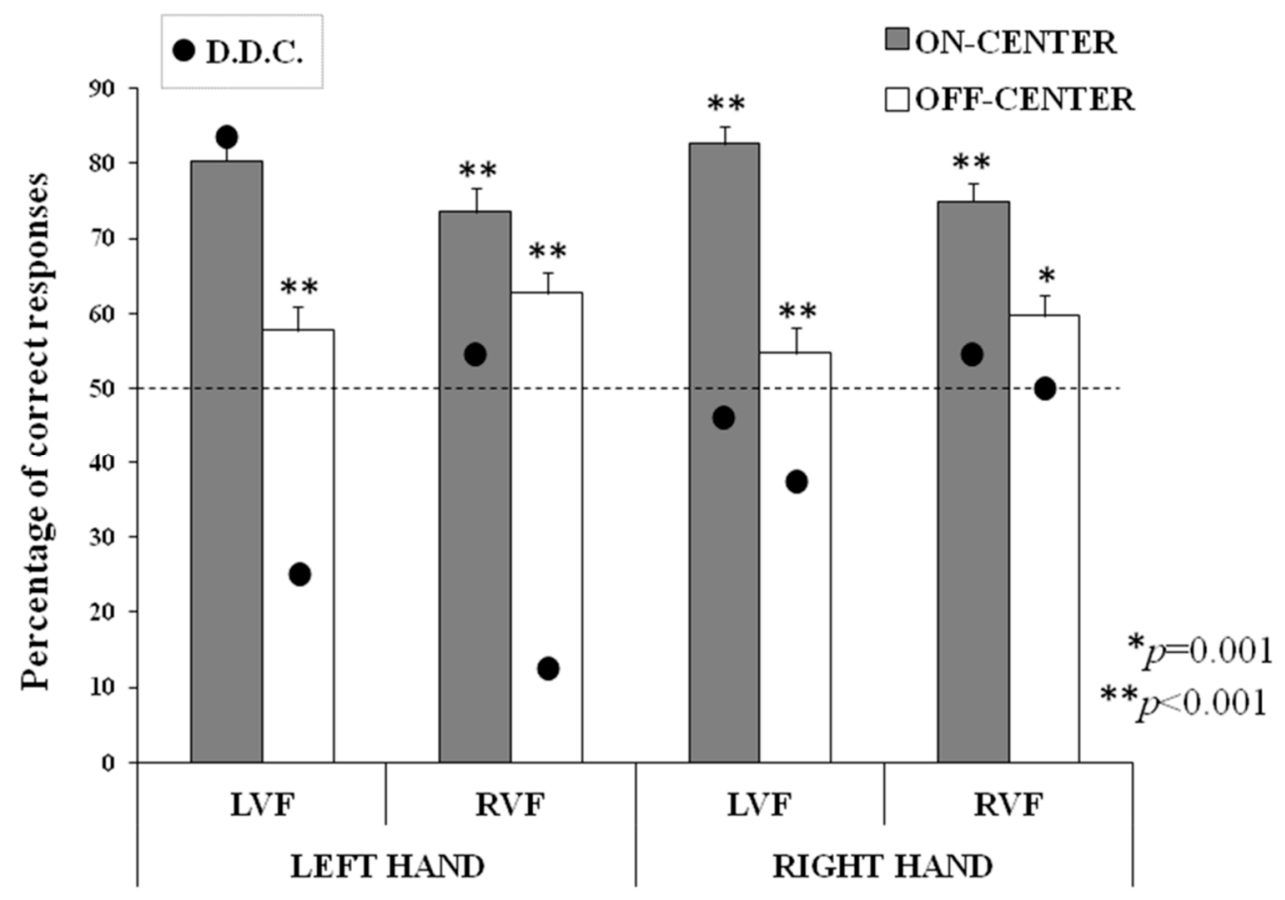

Figure 4. The percentage of correct responses in the left hand session (on the left) and in the right hand session (on the right), for On-Center stimuli (gray columns) and Off-Center stimuli (white columns) presented in the Left Visual Field (LVF) and in the Right Visual Field (RVF). The columns represent the results of healthy participants (bars represent standard errors), and black circles represent the results of D.D.C. Dashed lines represent chance levels (50\%). Asterisks show the significant comparisons between the performance of D.D.C. and healthy controls.

\section{Discussion}

The main aim of the present study was to investigate the possible hemispheric imbalance in the processing of visual symmetry for double-axes stimuli. As reviewed in the Introduction, a right-hemispheric superiority has been found in symmetry detection when mono-dimensional stimuli are presented, but no asymmetries have been found using bi-dimensional stimuli [24]. Nevertheless, we found that the right hemisphere is superior compared to the left hemisphere in the detection of bi-dimensional symmetry, both in healthy participants and in a callosotomized patient.

It has to be highlighted that in the sample of healthy participants, we did not find significant differences depending on the hand used to respond. Although, we did not hypothesize that the use of one hand would lead to statistical differences in the healthy sample, we did expect that in the patient with complete callosal section, the use of one hand would highlight the effects of the activity of the contralateral hemisphere. Indeed, in the split-brain literature the collection of responses given with one hand has been repeatedly exploited as a tool to test the contralateral hemispheric activity (e.g., [37-39]). We found that D.D.C.'s performance was at the chance level in all of the conditions when he provided the responses using his right hand, suggesting that the left disconnected hemisphere is not capable of discerning symmetry from asymmetry. Moreover, in the left hand session, the performance of the patient was below the chance level when off-center stimuli were presented, suggesting that the right disconnected hemisphere cannot correctly judge asymmetry or, alternatively, that a right-hemispheric bias for symmetry influences this result. A possible alternative explanation could be that the patient shows a simple response bias for on-center stimuli, but the fact that the percentage of correct responses for on-center stimuli presented in the LVF is $54 \%$ (random level), together with the fact that such a bias is present only when the left hand is used, makes this possibility less likely. Moreover, in the left hand session a main effect of symmetry confirmed that a "symmetry bias" is present in the right hemisphere (on-center stimuli were better categorized than off-center stimuli), for stimuli presented both in the 
LVF and in the RVF. Finally, the comparison between the performance of D.D.C. and the control group confirmed that only when the left hand was used and symmetric (on-center) stimuli were presented in the LVF was the comparison not significant, meaning that D.D.C. had the same accuracy level as the controls, whereas in all of the other conditions his performance was largely below that of the control group. Considered together, these results show that the right disconnected hemisphere of the split-brain patient can correctly process symmetry in bi-dimensional stimuli. The fact that the right (disconnected) hemisphere is superior to the left hemisphere in a number of tasks requiring spatial processing [12,13] is not a possible explanation for the present results. In fact, if it were the case, we should find a better performance in D.D.C. with both symmetrical and asymmetrical stimuli presented in the LVF. Similarly, a possible hemispheric difference in visual processing has to be discarded: by using the binocular rivalry paradigm, it has been shown, in two split-brain patients, that both hemispheres are able to process simple and complex stimuli (colored disks and faces), revealing a typical degree of binocular rivalry in both hemispheres [44-46]. Moreover, by means of binocular rivalry paradigms there is evidence of a redundancy effect [47], meaning that both hemispheres processed visual stimuli similarly and a callosal dysfunction can constitute an advantage in response times only when stimuli presented in both visual fields must be compared to each other [48]. The evidence of a right-hemispheric superiority which is specific for symmetry allows us to conclude that the symmetry bias is lateralized in the right hemisphere.

Regarding the healthy participants, the first crucial result found here is that they were better at categorizing symmetrical (on-center) rather than asymmetrical (off-center) stimuli, independently of the visual field of presentation. This evidence confirms the "symmetry bias" [23], that is the preference for symmetrical over asymmetrical configurations already proposed by the Gestalt theory. Some evidence has been collected concerning such a bias, showing that the human visual system detects symmetry more easily than asymmetry. First of all, symmetry detection is faster than asymmetry detection [49] and it affects the performance of observers even when it is not crucial for the task [50], as well as when the symmetrical arrangement constitutes the distracters during a visual search task [51]. Finally, it has been shown that symmetry is detected before eye movements are made towards a symmetric object, meaning that symmetry detection occurs also in the absence of overt attention [52]. This bias can have evolutionary roots, since symmetry is a relevant cue in the biological and physical world, so much so that it has been verified that it is innate and it is present in human infants (e.g., [53]), as well as in other species (e.g., [54]). The callosal hypothesis suggests that the symmetry bias could be due to the activity of two homologous areas in the two hemispheres, connected by means of the callosal fibers $[1,2,14-16]$. Starting from this view, it is predicted that when two patterns are presented in the two visual fields, they should be detected faster when they are symmetrical than when they are asymmetrical. Similarly, concerning split-brain patients, it can be expected that the absence of callosal connections should reveal no difference between the bilateral presentation of symmetrical or asymmetrical configurations. This prediction has been confirmed in split-brain and acallosal patients [55]. In the present study, we did not present stimuli bilaterally, and thus we did not aim at further exploring this issue, but we decided to present bi-dimensional stimuli in each visual field separately, in order to assess the specific propensity of each disconnected hemisphere at detecting bi-dimensional symmetry. To our knowledge, this is the first time a right-hemispheric superiority for bi-dimensional symmetry detection has been shown in a callosotomized patient. Moreover, the right-hemispheric bias for symmetry found in D.D.C. has been also confirmed in our control group: the results showed that when stimuli were presented in the LVF, the performance was better in the on-center than in the off-center condition, suggesting that the right hemisphere "prefers" symmetrical patterns also in healthy participants. The same conclusion might be suggested by the almost significant result showing a better discrimination of the on-center condition in the LVF than in the RVF. Moreover, a slightly better discrimination of the off-center condition in the RVF than in the LVF seems to suggest a complementary superiority in the two hemispheres in healthy brains, with a right-hemispheric 
preference for symmetry processing and an opposite left-hemispheric preference for asymmetry processing. Nevertheless, further investigations are needed in order to verify this hypothesis.

The right-hemispheric superiority in bi-dimensional symmetry detection are in accordance with the results by Verma et al. [56]. By presenting symmetric and asymmetric geometric figures in the periphery of each visual field, the authors found that both right-handed and left-handed participants with left-hemispheric speech dominance were more accurate when symmetrical stimuli were presented in the LVF (whereas contrasting cerebral asymmetries were found in participants with right-hemispheric dominance for speech). Differently from the results of the present study and from the results by Verma and colleagues [56], Wilkinson and Halligan [24] did not find a hemispheric imbalance in healthy participants, by using a divided visual field paradigm and double-axes stimuli. As reviewed above, however, we manipulated a number of parameters which could explain the difference in the results between the two studies, e.g., we lowered the presentation time of the stimuli. Also the eccentricity of the stimuli differed between the two studies, although it has been shown that this parameter did not influence symmetry detection [57]. Moreover, we tested a number of participants: Wilkinson and Halligan, in fact, divided their whole sample into different subgroups, each carrying out a different task. Thus, in that study 12 participants carried out the task with the double-axes stimuli (for more details on their study see the Introduction). Nevertheless, the results of the present study confirm those by Wilkinson and Halligan with single-axis stimuli; in fact, we found that the right hemisphere is superior to the left hemisphere in correctly judging symmetrical patterns. In single axis conditions, Wilkinson and Halligan exploited the "classical" bisection paradigm to assess symmetry perception, in which participants are asked to divide a line into two equal segments or to evaluate a pre-bisected line as composed of two symmetrical or asymmetrical segments. By using this paradigm, a number of studies confirmed the presence of "pseudo-neglect" in healthy participants (i.e., the systematic trend to bisect a line leftward than at its real center), and the opposite bias in patients suffering from neglect, who "ignore" the left hemispace and bisect the line rightmost rather than at the veridical center (for a review see [58]). A central issue in this context is the difference found in the performance of neglect patients according to the mono- or bi-dimensional stimuli they were required to bisect: in fact, if on one hand the rightward bisection is considered a "landmark" of hemispatial neglect, on the other hand, no such bias has been found when neglect patients were asked to find the central point of bi-dimensional stimuli. For instance, this dissociation has been evidenced by Halligan and Marshall [59] with a neglect patient: the patient showed the bias in horizontal and vertical line bisection, but he did not show biases when required to place a dot in the center of a square or of a circle. Conversely, however, MacDonald-Nethercott and colleagues [60] found the same magnitude of pseudoneglect (leftward bias) in healthy participants by using both lines and elliptical shapes.

A possible dissociation has been suggested between vertical and horizontal spatial processing: Churches and colleagues [61] have recently shown that independent of the shape of the bi-dimensional images, a consistent correlation exists between the biases within each dimension (vertical, horizontal) across different shapes, but that there is no correlation between the vertical and horizontal bias when the two dimensions are compared to one another. The authors concluded that the parietal ("where") route is involved in vertical plane processing, and that the occipital ("what") route is responsible for horizontal plane processing. This suggestion is in line with other studies, showing that the parietal cortex is mostly involved in line bisection (mono-dimensional stimuli), whereas the occipital extrastriate cortex is mostly involved in bi-dimensional stimuli activating the object-based route instead of the parietal space-based route (e.g., [62]). This is also a possible explanation for the dissociation found with neglect patients between mono- and bi-dimensional stimuli, in whom the spatial deficit is mainly due to a parietal or temporo-parietal lesion [58], and it is also confirmed by TMS evidence in healthy subjects. As reviewed above, in two different studies Bona and colleagues $[34,35]$ showed that a right-hemispheric stimulation disrupted symmetry detection in a stronger fashion than a left-hemispheric stimulation, when TMS was applied over the lateral occipital cortex. The involvement 
of the occipital cortex in multi-dimensional symmetrical patterns has been also confirmed in other fMRI and EEG studies [21,22].

We can conclude that the occipital, object-based visual route is responsible for the processing of bi-dimensional stimuli, and that this is also the cerebral substrate for symmetry detection when stimuli are bi-dimensional [36,62]. The present results confirm the "symmetry bias" consisting of the preference for symmetrical rather than asymmetrical configurations [23], and also show that such a bias is right-lateralized in the human brain. These speculations are based on the results we collected with healthy participants, but also-and importantly-with a patient with a complete section of the corpus callosum. This evidence is in line with other results collected before, by using different paradigms [60], and by exploiting both neuroimaging and electrophysiological measurements [21,22], as well as brain stimulation techniques $[34,35]$. We can also hypothesize that the same cerebral substrate could be the basis for the processing of three-dimensional stimuli, and thus for the detection of symmetry in the real world, but further work is needed in order to assess these hypotheses.

Acknowledgments: We thank very much D.D.C. for his willingness to collaborate in this study. We thank Gabriele Polonara for providing us with the MRI images of D.D.C., and Gabriella Venanzi for scheduling the patient's exams. Finally, we thank Elisabetta Conti and Margherita Davado, who helped us in recruiting and testing healthy participants.

Author Contributions: G.P. and L.T. conceived and designed the experiment; G.P., M.F., and N.F. performed the experiment; G.P. analyzed the data and wrote the paper; M.F. and L.T. revised the paper.

Conflicts of Interest: The authors declare no conflict of interest.

\section{References}

1. Tyler, C.W. Empirical aspects of symmetry perception. Spat. Vis. 1995, 9, 1-7. [CrossRef] [PubMed]

2. Wagemans, J. Detection of visual symmetries. Spat. Vis. 1995, 9, 9-32. [CrossRef] [PubMed]

3. Wagemans, J. Characteristics and models of human symmetry detection. Trends Cogn. Sci. 1997, 1, 346-352. [CrossRef]

4. Treder, M.S. Behind the looking glass: A review on human symmetry perception. Symmetry 2010, 2, 510-543. [CrossRef]

5. Van der Helm, P.A. Symmetry perception. In The Oxford Handbook of Perceptual Organization; Wagemans, J., Ed.; Oxford University Press: Oxford, UK, 2014.

6. Beck, D.M.; Pinsk, M.A.; Kastner, S. Symmetry perception in humans and macaques. Trends Cogn. Sci. 2005, 9, 405-406. [CrossRef] [PubMed]

7. Makin, A.D.; Wright, D.; Rampone, G.; Palumbo, L.; Guest, M.; Sheehan, R.; Cleaver, H.; Bertamini, M. An electrophysiological index of perceptual goodness. Cereb. Cortex 2016, 26, 4416-4434. [CrossRef] [PubMed]

8. Driver, L.; Baylis, G.C.; Rafal, R. Preserved figure-ground segregation and symmetry detection in visual neglect. Nature 1992, 360, 73-75. [PubMed]

9. Cattaneo, Z.; Fantino, M.; Silvanto, J.; Tinti, C.; Pascual-Leone, A.; Vecchi, T. Symmetry perception in the blind. Acta Psychol. 2010, 134, 398-402. [CrossRef] [PubMed]

10. Cattaneo, Z.; Bona, S.; Monegato, M.; Pece, A.; Vecchi, T.; Herbert, A.M.; Merabet, L.B. Visual symmetry perception in early onset monocular blindness. Visual Cogn. 2014, 22, 963-974. [CrossRef]

11. Prete, G.; Tommasi, L. Split-Brain Patients. In Encyclopedia of Evolutionary Psychological Science; Shackelford, T.K., Weekes-Shackelford, V.A., Eds.; Springer International Publishing AG: Cham (ZG), Switzerland, 2017; pp. 1-5.

12. Funnell, M.G.; Corballis, P.M.; Gazzaniga, M.S. A deficit in perceptual matching in the left hemisphere of a callosotomy patient. Neuropsychologia 1999, 37, 1143-1154. [CrossRef]

13. Corballis, P.M.; Funnell, M.G.; Gazzaniga, M.S. A dissociation between spatial and identity matching in callosotomy patients. Neuroreport 1999, 10, 2183-2187. [CrossRef] [PubMed]

14. Herbert, A.M.; Humphrey, G.K. Bilateral symmetry detection: Testing a 'callosal' hypothesis. Perception 1996, 25, 463-480. [CrossRef] [PubMed]

15. Corballis, M.C.; Beale, I.L. The Psychology of Left and Right; Lawrence Erlbaum: Oxford, UK, 1976; pp. x-227.

16. Braitenberg, V. Reading the structure of brains. Netw. Comput. Neural 1990,1,1-11. [CrossRef] 
17. Corballis, P.M. Visuospatial processing and the right-hemisphere interpreter. Brain Cogn. 2003, 53, 171-176. [CrossRef]

18. Wright, D.; Makin, A.D.J.; Bertamini, M. Electrophysiological responses to symmetry presented in the left or in the right visual hemifield. Coret $x$ 2017, 86, 93-108. [CrossRef] [PubMed]

19. Ban, H.; Yamamoto, H.; Fukunaga, M.; Nakagoshi, A.; Umeda, M.; Tanaka, C.; Ejima, Y. Toward a common circle: Interhemispheric contextual modulation in human early visual areas. J. Neurosci. 2006, 26, 8804-8809. [CrossRef] [PubMed]

20. Sasaki, Y.; Vanduffel, W.; Knutsen, T.; Tyler, C.; Tootell, R. Symmetry activates extrastriate visual cortex in human and nonhuman primates. Proc. Natl. Acad. Sci. USA 2005, 102, 3159-3163. [CrossRef] [PubMed]

21. Tyler, C.W.; Baseler, H.A.; Kontsevich, L.L.; Likova, L.T.; Wade, A.R.; Wandell, B.A. Predominantly extra-retinotopic cortical response to pattern symmetry. Neuroimage 2005, 24, 306-314. [CrossRef] [PubMed]

22. Palumbo, L.; Bertamini, M.; Makin, A. Scaling of the extrastriate neural response to symmetry. Vis. Res. 2015, 117, 1-8. [CrossRef] [PubMed]

23. Sasaki, Y. Processing local signals into global patterns. Curr. Opin. Neurobiol. 2007, 17, 132-139. [CrossRef] [PubMed]

24. Wilkinson, D.T.; Halligan, P.W. The effects of stimulus symmetry on landmark judgments in left and right visual fields. Neuropsychologia 2002, 40, 1045-1058. [CrossRef]

25. Breitmeyer, B.G. Simple reaction time as a measure of the temporal response properties of transient and sustained channels. Vis. Res. 1975, 15, 1411-1412. [CrossRef]

26. Prete, G.; Capotosto, P.; Zappasodi, F.; Laeng, B.; Tommasi, L. The cerebral correlates of subliminal emotions: An eleoencephalographic study with emotional hybrid faces. Eur. J. Neurosci. 2015, 42, 2952-2962. [CrossRef] [PubMed]

27. Prete, G.; D’ascenzo, S.; Laeng, B.; Fabri, M.; Foschi, N.; Tommasi, L. Conscious and unconscious processing of facial expressions: Evidence from two split-brain patients. J. Neuropsychol. 2015, 9, 45-63. [CrossRef] [PubMed]

28. Prete, G.; Laeng, B.; Fabri, M.; Foschi, N.; Tommasi, L. Right hemisphere or valence hypothesis, or both? The processing of hybrid faces in the intact and callosotomized brain. Neuropsychologia 2015, 68, 94-106. [CrossRef] [PubMed]

29. Wilkinson, D.T.; Halligan, P.W. Stimulus symmetry affects the bisection of figures but not lines: Evidence from event-related fMRI. NeuroImage 2003, 20, 1756-1764. [CrossRef] [PubMed]

30. Bertamini, M.; Makin, A.D. Brain activity in response to visual symmetry. Symmetry 2014, 6, 975-996. [CrossRef]

31. Wright, D.; Makin, A.D.; Bertamini, M. Right-lateralized alpha desynchronization during regularity discrimination: Hemispheric specialization or directed spatial attention? Psychophysiology 2015, 52, 638-647. [CrossRef] [PubMed]

32. Makin, A.D.; Rampone, G.; Wright, A.; Martinovic, J.; Bertamini, M. Visual symmetry in objects and gaps. J. Vis. 2014, 14, 1-12. [CrossRef] [PubMed]

33. Makin, A.D.; Rampone, G.; Pecchinenda, A.; Bertamini, M. Electrophysiological responses to visuospatial regularity. Psychophysiology 2013, 50, 1045-1055. [CrossRef] [PubMed]

34. Bona, S.; Herbert, A.; Toneatto, C.; Silvanto, J.; Cattaneo, Z. The causal role of the lateral occipital complex in visual mirror symmetry detection and grouping: An fMRI-guided TMS study. Cortex 2014, 51, 46-55. [CrossRef] [PubMed]

35. Bona, S.; Cattaneo, Z.; Silvanto, J. The causal role of the occipital face area (OFA) and lateral occipital (LO) cortex in symmetry perception. J. Neurosci. 2015, 35, 731-738. [CrossRef] [PubMed]

36. Cattaneo, Z.; Mattavelli, G.; Papagno, C.; Herbert, A.; Silvanto, J. The role of the human extrastriate visual cortex in mirror symmetry discrimination: A TMS-adaptation study. Brain Cogn. 2011, 77, 120-127. [CrossRef] [PubMed]

37. Gazzaniga, M.S. The split brain in man. Sci. Am. 1967, 217, 24-29. [CrossRef] [PubMed]

38. Levy, J.; Trevarthen, C.; Sperry, R.W. Perception of bilateral chimeric figures following hemispheric deconnexion. Brain 1972, 95, 61-78. [CrossRef] [PubMed]

39. Prete, G.; Fabri, M.; Foschi, N.; Tommasi, L. Geometry, landmarks and the cerebral hemispheres: 2D spatial reorientation in split-brain patients. J. Neuropsychol. 2016. [CrossRef] [PubMed]

40. Corballis, M.C.; Birse, K.; Paggi, A.; Manzoni, T.; Pierpaoli, C.; Fabri, M. Mirror-image discrimination and reversal in the disconnected hemispheres. Neuropsychologia 2010, 48, 1664-1669. [CrossRef] [PubMed] 
41. Fabri, M.; Polonara, G.; Mascioli, G.; Paggi, A.; Salvolini, U.; Manzoni, T. Contribution of the corpus callosum to bilateral representation of the trunk midline in the human brain: An fMRI study of callosotomized patients. Eur. J. Neurosci. 2006, 23, 3139-3148. [CrossRef] [PubMed]

42. Oldfield, R.C. The assessment and analysis of handedness: The Edinburgh Inventory. Neuropsychologia 1971, 9,97-114. [CrossRef]

43. Prete, G.; Fabri, M.; Foschi, N.; Brancucci, A.; Tommasi, L. The "consonance effect" and the hemispheres: A study on a split-brain patient. Laterality 2015, 20, 257-269. [CrossRef] [PubMed]

44. O'shea, R.P.; Corballis, P.M. Binocular rivalry between complex stimuli in split-brain observers. Brain Mind 2001, 2, 151-160. [CrossRef]

45. O'Shea, R.P.; Corballis, P.M. Binocular rivalry in split-brain observers. J. Vis. 2003, 3, 610-615. [CrossRef] [PubMed]

46. Miller, J. Exaggerated redundancy gain in the split brain: A hemispheric coactivation account. Cogn. Psychol. 2004, 49, 118-154. [CrossRef] [PubMed]

47. Ritchie, K.L.; Bannerman, R.L.; Turk, D.J.; Sahraie, A. Eye rivalry and object rivalry in the intact and split-brain. Vis. Res. 2013, 91, 102-107. [CrossRef] [PubMed]

48. Ritchie, K.L.; Bannerman, R.L.; Sahraie, A. Redundancy gain in binocular rivalry. Perception 2014, 43, 1316-1328. [CrossRef] [PubMed]

49. Wagemans, J.; Van Gool, L.; D'ydewalle, G. Detection of symmetry in tachistoscopically presented dot patterns: Effects of multiple axes and skewing. Atten. Percept. Psychophys. 1991, 50, 413-427. [CrossRef]

50. Van der Helm, P.A.; Treder, M.S. Detection of (anti) symmetry and (anti) repetition: Perceptual mechanisms versus cognitive strategies. Vis. Res. 2009, 49, 2754-2763. [CrossRef] [PubMed]

51. Wolfe, J.M.; Friedman-Hill, S.R. On the role of symmetry in visual search. Psychol. Sci. 1992, 3, $194-198$. [CrossRef]

52. Kootstra, G.; de Boer, B.; Schomaker, L.R. Predicting eye fixations on complex visual stimuli using local symmetry. Cog. Comp. 2011, 3, 223-240. [CrossRef] [PubMed]

53. Bornstein, M.H.; Ferdinandsen, K.; Gross, C.G. Perception of symmetry in infancy. Dev. Psychol. 1981, 17, 82. [CrossRef]

54. Mascalzoni, E.; Osorio, D.; Regolin, L.; Vallortigara, G. Symmetry perception by poultry chicks and its implications for three-dimensional object recognition. Proc. R. Soc. Lond. B Biol. Sci. 2011, 279, 841-846. [CrossRef] [PubMed]

55. Roser, M.; Corballis, M.C. Interhemispheric neural summation in the split brain with symmetrical and asymmetrical displays. Neuropsychologia 2002, 40, 1300-1312. [CrossRef]

56. Verma, A.; Van der Haegen, L.; Brysbaert, M. Symmetry detection in typically and atypically speech lateralized individuals: A visual half-field study. Neuropsychologia 2013, 51, 2611-2619. [CrossRef] [PubMed]

57. Rampone, G.; O'Sullivan, N.; Bertamini, M. The Role of Visual Eccentricity on Preference for Abstract Symmetry. PLoS ONE 2016, 11, e0154428. [CrossRef] [PubMed]

58. Karnath, H.O.; Rorden, C. The anatomy of spatial neglect. Neuropsychologia 2012, 50, 1010-1017. [CrossRef] [PubMed]

59. Halligan, P.W.; Marshall, J.C. Figural modulation of visuo-spatial neglect: A case study. Neuropsychologia 1991, 29, 619-628. [CrossRef]

60. Macdonald-Nethercott, E.M.; Kinnear, P.R.; Venneri, A. Bisection of shapes and lines: Analysis of the visual and motor aspects of pseudoneglect. Percept. Mot. Skills 2000, 91, 217-226. [CrossRef] [PubMed]

61. Churches, O.; Loetscher, T.; Thomas, N.A.; Nicholls, M.E. Perceptual biases in the horizontal and vertical dimensions are driven by separate cognitive mechanisms. Q. J. Exp. Psychol. 2017, 70, 444-460. [CrossRef] [PubMed]

62. Fink, G.R.; Marshall, J.C.; Weiss, P.H.; Shah, N.J.; Toni, I.; Halligan, P.W.; Zilles, K. 'Where' depends on 'what': A differential functional anatomy for position discrimination in one-versus two-dimensions. Neuropsychologia 2000, 38, 1741-1748. [CrossRef]

(C) 2017 by the authors. Licensee MDPI, Basel, Switzerland. This article is an open access article distributed under the terms and conditions of the Creative Commons Attribution (CC BY) license (http:/ / creativecommons.org/licenses/by/4.0/). 